\title{
Overnight Risk Model: A Unique Capability
}

\author{
Abigail Hsu ${ }^{1}$, Ryan Kaufman ${ }^{1}$, Hyunkyung $\operatorname{Lim}^{1} \&$ James Glimm ${ }^{1}$ \\ ${ }^{1}$ Department of Applied Mathematics and Statistics, Stony Brook University, New York, USA \\ Correspondence: Abigail Hsu, Department of Applied Mathematics and Statistics, Stony Brook University, New York, \\ USA.
}

Received: August 25, 2020

Accepted: September 23, $2020 \quad$ Available online: September 30, 2020

doi:10.11114/aef.v7i6.4981

URL: https://doi.org/10.11114/aef.v7i6.4981

\begin{abstract}
We present a novel risk measurement model capable of capturing overnight risk i.e. the risk encountered between the closing time of the previous day and the opening time of the next day. The risk model captures both the overnight risk and also the intraday risk. Statistical models of intraday asset returns must separate the market opening period from the remainder of the day as these follow statistical laws with different properties. Here we present results showing our two models for these two distinct periods.
\end{abstract}

Keywords: risk measurement, intraday, overnight

\section{Introduction}

The closing price for a share of stock is the last price paid before market close at the end of its normal business hours. The opening price is the first price paid during the next business day. The risk associated with the price jumps from market closing to the following market opening is about half of the total intraday risk. The 30 minutes following market opening are characterized by rapid price fluctuations and can be described as a period of price discovery. The ability to model this half of the total daily risk appears to be a novel and important contribution to risk modeling. The intraday trading after the first thirty minutes of the day follows our standard risk analysis algorithms. The algorithm is of value to high frequency traders as an aid in assessing slippage costs in exiting a trading position at the end of the holding period. We do not model this price discovery period.

\section{Literature Review}

The general framework of ideas seems to be known by the trading community. Intraday risk has been considered by a number of authors. The anomalies on weekend, holiday, turn of the month, and intraday effects have been reviewed by Thaler (Thaler, 1987). A framework for analyzing the dynamic effects on intraday risk premia has been presented (Spiegel \& Subrahmanyam, 1995). The intraday periodicity in the return volatility has been explored (Anderson and Bollerslev, 1998). (Bollen \& Inder, 2002) proposed a new approach on estimating the daily volatility from intraday data. (Giot, 2011) applied multiple market risk modes to compare their performance for intraday returns data for stocks traded on the New York Stock Exchange.

Our risk model is documented to be successful and has accuracy for example for CVaR at the $99 \%$ confidence level for 10 and 30 minutes intraday and overnight data. The intraday risk can be evaluated efficiently by using analytic methods without resorting to a large number of Monte Carlo simulations. The overnight risk is known qualitatively to the trading community, but it seems that a carefully reasoned, systematic and quantitative risk model has not been developed. In this sense, we believe that the analysis we present here is unique.

\section{The Risk Model}

Instead of using tick-by-tick data, we aggregate the intraday price increments over time intervals of equal length to minimize microstructure noise. We transform the price increments into returns and apply a model that can account for the observed characteristics: auto-correlation, clustering of volatility, long-range dependence, and heavy tails. To describe these empirical phenomena, this model removes volatility clustering and models the heavy tails of the innovations.

In addition to its flexibility in modeling financial time series and distributional properties, the model allows for multi-step scenario generation. This feature is useful when forecasting risk overnight between the closing time of the 
previous day and the opening time of the next day.

The overnight risk is estimated with the Monte Carlo method. The model parameters are estimated using a rolling time window of intraday data, i.e. with overnight returns removed. Possible future scenarios are generated representing the expected return for the next $\mathrm{n}$ steps $(\mathrm{n} \geq 1)$, with the size of a step equal to the frequency of the input data. The optimal number of overnight steps depends on the time frequency of data and is approximately half of the number of full day observation periods, a value consistent with the observation that the overnight risk is about half of the full day risk. For intraday risk calculations, we use the normal single step prediction model. For this reason, we do not correctly model the price discovery at opening for periods below 30 minutes. For the next step following the opening, we use the same historical data as the ones used in the overnight prediction. Since the only new data available would be the overnight return, which is not included as a model input. However, this intraday prediction is for only a single step.

On the basis of the generated scenarios, risk is calculated using the industry-standard risk measures: value-at-risk (VaR) and conditional value-at-risk (CVaR), also known as expected shortfall (ES). Value-at-risk (VaR) is defined as the negative of a low quantile in the left tail of the return distribution. The standard choice for the quantile probability level is $1 \%$ or $5 \%$. Because VaR provides no information regarding losses beyond the VaR threshold, the risk model also calculates CVaR. CVaR is defined as the average loss provided that the loss is larger than a given VaR level. Risk reserve requirements are formulated in Basel II based on VaR (Basel committee on Banking Supervision, 2004) and in Basel III based on CVaR (Basel committee on Banking Supervision, 2011). Overnight risk, not normally considered, is a significant $(50 \%)$ contributor to the total daily risk, meaning that liquidity reserves for overnight should be high enough to accommodate this enhanced level of risk.

\section{Backtesting the Model}

A risk model is backtested to determine whether the risk forecast is accurate within the limits imposed by the finite sample size of the backtesting sample. For example, if the forecasted VaR's at $99 \%$ confidence levels are correct, then the expected number of time periods in which the realized loss exceeds the forecasted VaR should be approximately $1 \%$ of the time periods in the backtesting time window. The backtesting conducted follow the outline proposed (Campbell, 2005). When realized losses exceed the forecasted VaR, we have a case of exceedance. Thus, we have a probability estimate describing the number of exceedances for a statistically valid risk model. Predictions from the risk model of Section 3 are tested against observed data. The number of exceedances is then assessed by a binomial model as too few, too many, or satisfactory. Thus, we generate confidence values (p-values) for the deviation of the number of exceedances from its expected mean value according to the $99 \%$ confidence value. This is the intuition behind the standard test in the financial industry known as Kupiec's test. The p-value of the expected shortfall is defined, and conclusive results are achieved (Acerbi \& Székely, 2017).

The quantile-quantile plot allows us to assess the ability of the risk model to accurately describe the risk associated with all quantiles of observed and predicted returns. The Kolmogorov-Smirnov test statistic, D, assesses the prediction errors across all quantiles in the $\mathrm{L}^{\infty}$ sense. See Tables 1 and 2 for color coding schemes for p-values and statistics. Small values of the Kolmogorov-Smirnov (KS) statistic are better. We do not assess confidence values for the KS statistic, so the labeling in Table 2 is arbitrary.

Table 1. Color-coding scheme for p-values

\begin{tabular}{|l|l|l|l|}
\hline $\mathrm{p}$-values ( $\mathrm{p})$ & $\mathrm{p}<0.01$ & $0.01 \leq \mathrm{p}<0.05$ & $\mathrm{p} \geq 0.5$ \\
\hline color (risk underestimation) & & & \\
\hline color (risk overestimation) & & & \\
\hline
\end{tabular}

Description: The color-coding scheme for p-values of statistical analysis (larger is better).

Table 2. Color-coding scheme for the Kolmogorov-Smirnov test statistic

\begin{tabular}{|l|l|l|l|}
\hline Kolmogorov-Smirnov statistic (D) & $\mathrm{D}>0.12$ & $0.06<\mathrm{D} \leq 0.12$ & $\mathrm{D} \leq 0.06$ \\
\hline color & & & \\
\hline
\end{tabular}

Description: The color-coding scheme for the Kolmogorov-Smirnov test statistic (smaller is better).

\section{Data}

We test the model on SPY data for the years 2018 and 2019 for the 10 and 30 minute frequency data. We analyze 
separately the risk of the opening time and the intraday risk. We show results for 10 and 30 minute frequencies.

\section{Backtesting Results}

Shares of SPY (SPDR S\&P 500 ETF Trust) are traded on the NYSE, which usually opens at 9:30am and closes at 4:00pm. There are then two separate problems when measuring intraday risk. The first is the risk of any 30-minute time interval intraday between 9:30am and 4:00pm and the second is the risk of overnight. That is, if there are open positions at closing, the overnight risk model determines the risk for the following market opening. It is also clear that the information flow overnight is reduced and therefore the overnight risk is neither determined by the elapsed overnight period nor by a single intraday period. It is approximately equal to half of the total intraday risk, that is 3.5 hours, or a about $1 / 6$ of the overnight period.

\subsection{SPY 10 Minute Frequency Return Analysis}

We present the SPY 10 minute frequency analysis for intraday risk and overnight risk separately. The comparisons of VaR to the corresponding real returns are shown in Figure 1. Similarly, Figure 2 presents the comparison using the quantile-quantile plot. Table 3 displays the p-values and Kolmogorov-Smirnov test statistic for intraday risk and overnight risk.
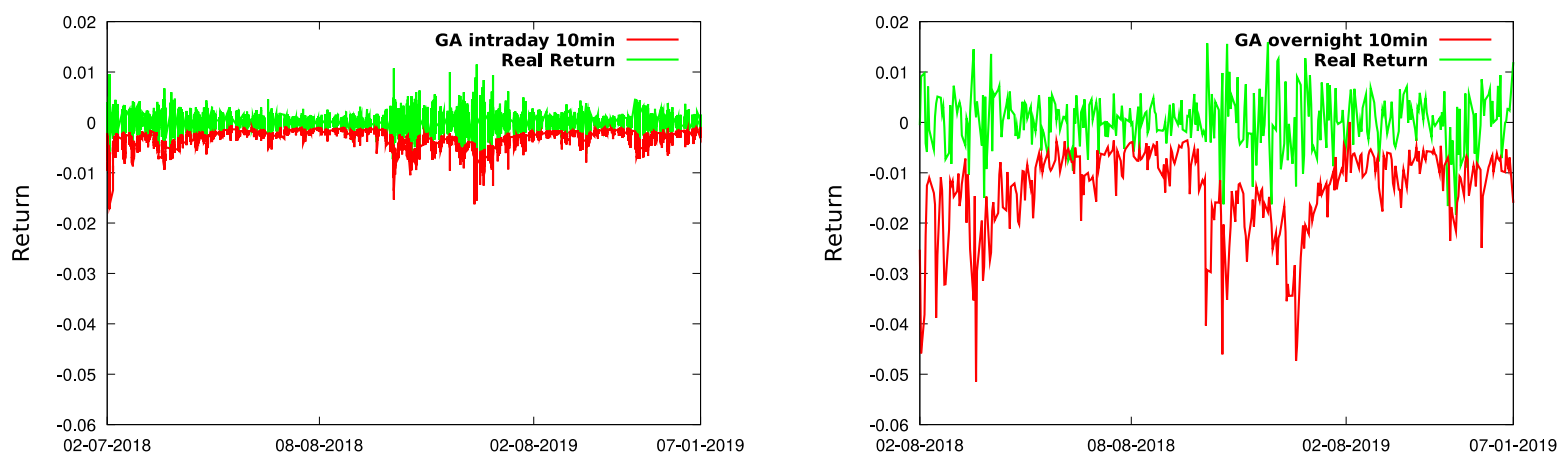

Figure $1 . \mathrm{VaR}$ at $99 \%$ for 10 minute

Description: Realized 10 minute returns together with the forecasted VaR at $99 \%$ confidence value using our risk models for intraday (left) and overnight(right).
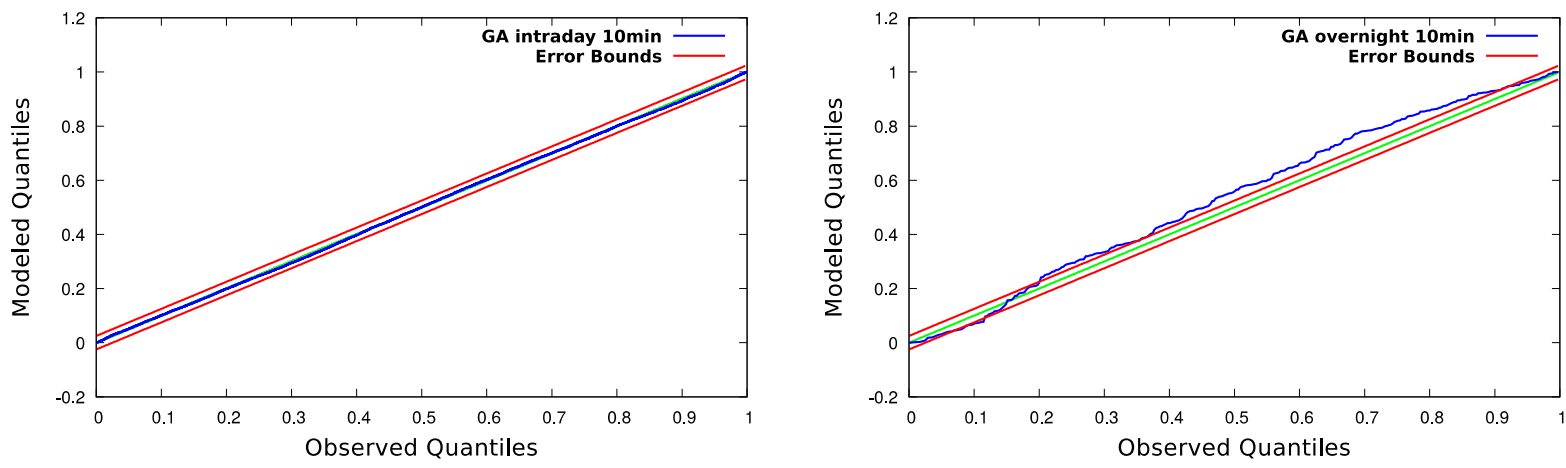

Figure 2. QQ plot for 10 minute

Description: A quantile-quantile plot of our risk model versus data for 10 minute frequency for intraday (left) and overnight (right).

Table 3. The statistical assessment for 10 minute frequency data

\begin{tabular}{|l|l|l|l|l|}
\hline Periods & Exceedances & Kupiec p-values & ES p-values & KS stat \\
\hline Intraday & $139 / 13632$ & 0.82 & 0.216 & 0.0074 \\
\hline Overnight & $10 / 350$ & 0.045 & 0.075 & 0.088 \\
\hline
\end{tabular}

Description: The second to fifth columns show the number of exceedances, Kupiec's test p-values, expected shortfall p-values at 99\% confidence level. The last column is the Kolmogorov-Smirnov test statistic for all quantiles. 


\subsection{SPY 30 Minute Frequency Return Analysis}

We present the SPY 30 minute frequency analysis for intraday risk and overnight risk separately. The comparisons of VaR to the corresponding real returns are shown in Figure 3. In addition, Figure 4 presents the comparison using the quantile-quantile plot for 30 minute frequency data. The p-values and Kolmogorov-Smirnov test statistic for intraday risk and overnight risk is also shown in Table 4.
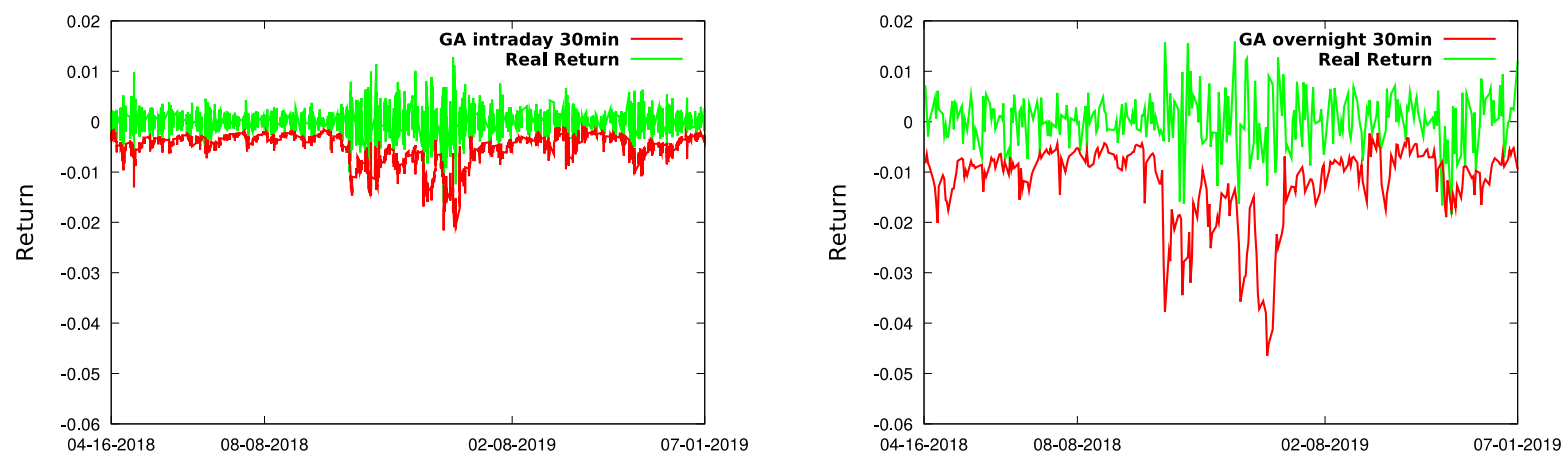

Figure 3. VaR at $99 \%$ for 30-minute frequency data

Description: Realized 30 minute returns together with the forecasted VaR at $99 \%$ confidence value using our risk models for intraday (left) and overnight(right).
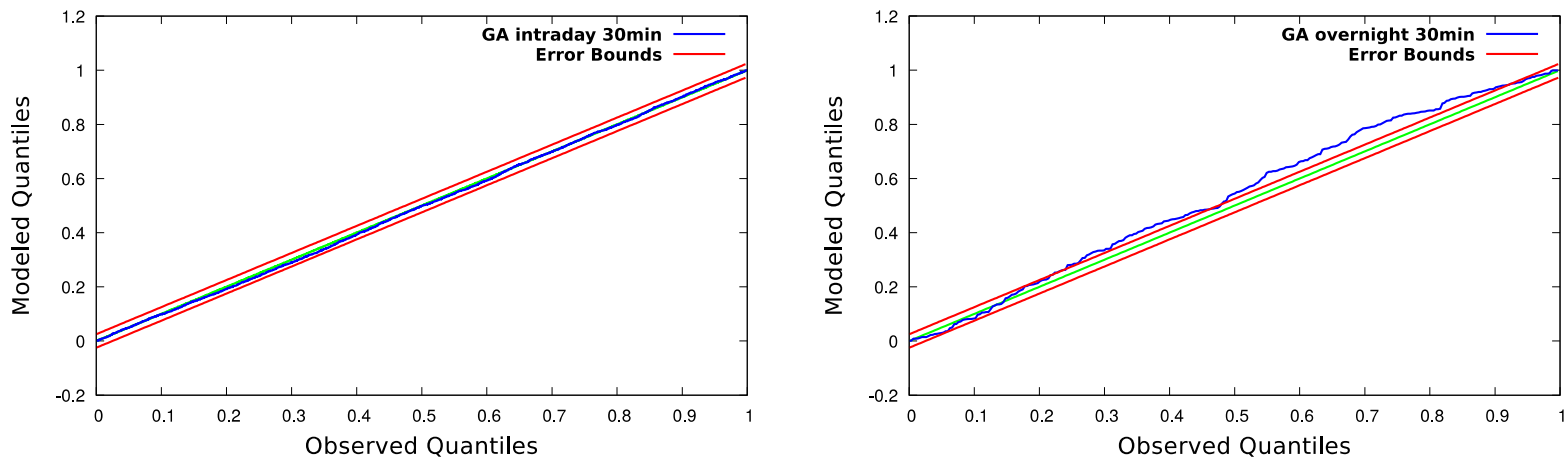

Figure 4. QQ plot for 30-minute frequency data

Description: A quantile-quantile plot of the risk model versus data for 30 minute frequency for intraday (left) and overnight (right).

Table 4. The statistical assessment for 30 minute frequency data

\begin{tabular}{|l|l|l|l|l|}
\hline Periods & Exceedances & Kupiec p-values & ES p-values & KS stat \\
\hline Intraday & $43 / 3938$ & 0.569 & 0.433 & 0.0135 \\
\hline Overnight & $5 / 304$ & 0.304 & 0.569 & 0.09 \\
\hline
\end{tabular}

\section{Summary}

We present a unique model for intraday and overnight risk modeling at 10 and 30 minute frequency. We demonstrate the accuracy of the model by backtesting, both for the overnight risk and the risk throughout the trading day. Backtesting includes VaR, CVaR (estimated shortfall), the Kolmogorov-Smirnov statistic and quantile-quantile (QQ) plots and confirms the accuracy of our risk model.

The model assesses reserve requirements throughout the day and especially for the overnight period. The optimal number of overnight steps has been determined approximately half of the number of full day observation periods. It is useful in high speed trading in assessing the market slippage costs when exiting a position.

\section{Future Developments}

A market price discovery algorithm would aid in the modeling of shorter time intraday statistics, especially during 
market opening, which is characterized by high volatility and low volume. A volatility related subordinator would improve the modeling of intraday volatility spikes and would improve the modeling of the market opening period. A simple and common set of risk model statistics to assess the data were employed. A full treatment of the many possible criteria for statistical attributes of the solutions is a separate issue, and for this reason, while not considered here, is an intended topic of further research. The risk models used here are based on standard ideas that address well understood features of asset returns: heavy tails, volatility clustering and long-range dependence. These ideas will be explored further in a separate paper.

\section{References}

Acerbi, C., \& Székely, B. (2017). General Properties of Backtestable Statistics. SSRN Electronic Journal. https://doi.org/10.2139/ssrn.2905109

Anderson, T., \& Bollerslev, T. (1998). Intraday periodicity and volatility persistence in financial markets. Journal of Empirical Finance. Retrieved from https://www.sciencedirect.com/science/article/pii/S0927539897000042

Basel committee on Banking Supervision. (2004). International convergence of capital measurement and capital standards. Technical report. Retrieved from https://www.bis.org/publ/bcbs107.htm

Basel committee on Banking Supervision. (2011). A global regulatory framework for more resilient banks and banking systems. Technical report. Retrieved from https://www.bis.org/publ/bcbs189.htm

Bollen, B., \& Inder, B. (2002). Estimating daily volatility in financial markets utilizing intraday data. Journal of Empirical Finance. https://doi.org/10.1016/S0927-5398(02)00010-5

Campbell, S. (2005). A review of backtesting and backtesting procedures. Journal of Risk. https://doi.org/10.17016/FEDS.2005.21

Pierre, G. (2005). Market risk models for intraday data. The European Journal of Finance, 11(4), 309-324. https://doi.org/10.1080/1351847032000143396

Spiegel, M., \& Subrahmanyam, A. (1995). On Intraday Risk Premia. The Journal of Finance, 50, 319-339. https://doi.org/10.1111/j.1540-6261.1995.tb05176.x

Thaler, H. (1987). Anomalies: Weekend, Holiday, Turn of the Month, and Intraday Effects. Journal of Economic Perspectives, 1(2), 169-177. https://doi.org/10.1257/jep.1.2.169

\section{Copyrights}

Copyright for this article is retained by the author(s), with first publication rights granted to the journal.

This is an open-access article distributed under the terms and conditions of the Creative Commons Attribution license which permits unrestricted use, distribution, and reproduction in any medium, provided the original work is properly cited. 